\title{
Ras1 signaling and transcriptional competence in the R7 cell of Drosophila
}

\author{
Rachele C. Kauffmann, Songhui Li, Paulette A. Gallagher, Jianjun Zhang, and Richard W. Carthew ${ }^{1}$ \\ Department of Biological Sciences, University of Pittsburgh, Pittsburgh, Pennsylvania 15260 USA
}

\begin{abstract}
The receptor tyrosine kinase Sevenless determines $R 7$ cell fate by activation of the Ras 1 pathway in a subset of equivalent cells competent to respond in the Drosophila eye. We show that the prospero gene becomes transcriptionally activated at a low level in all Sevenless-competent cells prior to Sevenless signaling, and this requires the activities of Ras1 and two Ras1/MAP kinase-responsive ETS transcription factors. Restriction of high-level prospero expression to the $\mathbf{R} 7$ cell appears as a subsequent event, which requires Sevenless activation of the Ras1/MAP kinase pathway. We show that Phyllopod, a nuclear factor whose expression is induced by Sevenless, interacts with another nuclear factor, Sina, to form a complex, and that both factors are involved in upregulating transcription of the prospero gene in the eye. Ultimately, prospero expression is required for proper connectivity of $R 7$ photoreceptor axons to their synaptic targets. Our results suggest that specific transcriptional responses are linked to the mode of activation of the Ras1/MAP kinase signal transduction pathway.
\end{abstract}

[Key Words: Drosophila; prospero; sevenless; Ras1; eye development]

Received May 29, 1996; revised version accepted July 11, 1996.

Receptor tyrosine kinases (RTKs) are involved in signaling between cells to regulate proliferation and differentiation. RTK signaling can be substituted by gain-offunction variants of Ras, MEK, and MAP kinase (MAPK), all of which are components of the same signal transduction pathway (for review, see Zipursky and Rubin 1994; Marshall 1995). This suggests that Ras-mediated activation of MAPK is a critical event in signal transduction from RTKs. Paradoxically, the Ras pathway is commonly required in many cell types during development, and yet activation of distinct RTKs generates different cellular responses. A central issue in understanding RTK signaling is how a cell-type specific response is generated by use of a common Ras pathway. In rat $\mathrm{PC} 12$ cells, the decision to differentiate into a neuron or to proliferate is guided by the duration of MAPK activation /Marshall 1995). In turn, this is regulated by the specific ligandRTK interaction triggered in PC12 cells. In the Drosophila eye, specific responses are in part determined by cell-specific factors that function downstream of MAPK (Dickson 1995). Here we describe a gene, prospero (pros), that is necessary for cell-type specific differentiation in the Drosophila eye and is transcriptionally regulated by multiple signals that activate the same Rasl pathway.

The Drosophila eye develops by the reiterative patterning of $\sim 800$ ommatidia initiated in the larval eye imaginal disc. Differentiation occurs progressively from posterior to anterior across the eye disc, with the leading edge of differentiation marked by an indentation called

\footnotetext{
${ }^{1}$ Corresponding author.
}

the morphogenetic furrow. Assembly of each ommatidium behind the furrow occurs by the sequential recruitment of undifferentiated cells into distinct developmental programs (Zipursky and Rubin 1994). The R7 photoreceptor cell is the last photoreceptor to be recruited into the ommatidial cluster, and is followed by recruitment of four non-neuronal cone cells. Although only one R7 cell develops in each ommatidium, there is a potential for five cells to develop with an R7 cell identity. These cells include the R7 and cone cell precursors and are collectively known as the R7 equivalence group. Determination of $\mathrm{R} 7$ cell fate is dependent upon signaling by the Sevenless (Sev) RTK in response to an inductive interaction with the neighboring $\mathrm{R} 8$ cell. This is transduced within the presumptive $\mathrm{R} 7$ cell by a cascade of proteins including Ras1, Raf, and Rolled/MAPK. All of the cells within the $\mathrm{R} 7$ equivalence group can be transformed to an $\mathrm{R} 7$ cell fate by activation at any point along the sev/Ras1/rolled signaling pathway (Basler et al. 1991; Fortini et al. 1992; Brunner et al. 1994b). Conversely, genetic disruption of the pathway results in adoption of a cone cell fate (Tomlinson and Ready 1987; Simon et al. 1991; Biggs et al. 1994).

Biochemical studies of vertebrate MAPK have shown that activated MAPK translocates to the nucleus where it phosphorylates different transcription factors (Marshall 1995). Nuclear target molecules of activated rolled/ MAPK in the R7 equivalence group include the ETSdomain transcription factors, Yan and Pointed (Pnt). A variety of studies support a model in which the balance between the activities of Pnt and Yan determine whether a cell adopts a cone or R7 cell fate (Lai and Rubin 1992; 
O'Neill et al. 1994; Brunner et al. 1994a; Rebay and Rubin 1995/. When uninduced, Yan and Pnt are unphosphorylated, with Yan acting as a repressor of target gene expression. Upon induction, Yan and Pnt are phosphorylated, resulting in inhibition of Yan's repression and stimulation of target gene expression by Pnt.

The phyllopod (phyl) gene is a possible target gene of the Rasl pathway /Chang et al. 1995; Dickson et al. 1995). It is expressed in the R1, R6, and R7 precursor cells where it functions to promote their determination as photoreceptor cells. In the absence of phyl, they are transformed into cone cells. Misexpression of phyl in cone cell precursors transforms their fate to an R7 cell provided the sina gene is present. This is consistent with the demonstrated requirements for sina in normal R7 determination (Carthew and Rubin 1990). Both phyl and sina encode nuclear factors of unknown biochemical function.

These studies have identified a number of nuclear factors that play important roles in $\mathrm{R} 7$ determination. However, target genes of the factors that are specifically expressed in $\mathrm{R} 7$ cells and are required for $\mathrm{R} 7$ differentiation have not been identified. With the aim of identifying genes that function downstream of $\sin a$, we performed a genetic screen for mutations that dominantly enhance a mutant sina phenotype. One of the genes we identified, pros, has been shown previously to encode a putative transcription factor required for differentiation of the embryonic nervous system (Doe et al. 1991; Vaessin et al. 1991). We show that pros is required in the eye for proper connectivity of R7 photoreceptor axons to their synaptic targets in the brain. We also show that pros transcription is initially activated in all cells of the R7 equivalence group by activation of the Ras 1 pathway. Yan and Pnt proteins appear to mediate these effects. Later, R7 cells specifically transcribe pros at higher levels in response to Sev activation of Ras1. Yan and Pnt are also intermediates of the upregulation of transcription. In addition, Phyl is involved in transcriptional upregulation of pros, albeit in a functionally redundant capacity. We show that sina is required for phyl's effect on pros transcription and that phyl protein forms a specific complex with sina protein both in vivo and in vitro. These results imply that pros transcription is regulated by Ras 1 through two different mechanisms, one that initiates transcription in the R7 equivalence group and one that stimulates increased levels of transcription in the R7 cell.

\section{Results}

pros is a dominant enhancer of sina

Loss of sina activity in the $\mathrm{R} 7$ precursor cell leads to its transformation to a cone cell. In flies homozygous for a weak loss-of-function allele $\left(\sin a^{4}\right), \sim 20 \% \quad(n=660)$ of the ommatidia are missing the $\mathrm{R} 7$ photoreceptor cell (Fig. 1A). To identify genes that interact with sina, we screened a collection of lethal P-element enhancer trap insertions for loss-of-function mutations that dominantly enhance or suppress the sin $a^{4}$ phenotype. From this screen, we identified an allele of pros, 1(3)10419, which enhanced the sin $a^{4}$ phenotype, increasing the number of R7-less ommatidia to $50 \%(n=316$; Fig. 1B). A transcript-null allele of pros, pros ${ }^{\prime O 1}$ (Vaessin et al. 1991), was tested for its ability to dominantly enhance the sina $a^{4}$ phenotype. Like the pros allele $1(3) 10419$ (pros $^{10419}$ ), pros $^{101}$ increased the number of R7-less ommatidia to $50 \%(n=1420$; Fig. $1 C)$. To demonstrate that a reduction in pros product was responsible for the enhanced phenotype, we increased the amount of Pros by generating transgenic flies in which a pros cDNA was put under the control of the sev enhancer (sev-pros). This enhancer is active in photoreceptor cells R3, R4, R7, the cone cell precursors, and (at low levels) in R1 and R6 (Zipursky and Rubin 1994). The enhancement of the $\sin a^{4}$ phenotype was not observed in flies bearing one copy of the sev-pros transgene in a $\sin a^{4} / \sin a^{4} ; p r o s^{101} /+$ background ( $n=1859$; Fig. 1D). Therefore, reducing the dosage of the pros gene enhances the R7 cell defect caused by $\sin a^{4}$.

\section{pros is required for $R 7$ axon targeting}

We wished to determine the homozygous mutant pros phenotype to further understand the function of pros in eye development. Since both $\operatorname{pros}^{10419}$ and pros ${ }^{101}$ are homozygous lethal, we generated pros ${ }^{101}$ mutant clones in a wild-type eye (Fig. 1E). Within the pros ${ }^{-}$clones, $23 \%(n=215)$ of $\mathrm{R} 7$ cells had an abnormal morphology, as evidenced by uncharacteristically small light-sensing organelles or rhabdomeres. We also examined the synaptic connections of pros $^{-}$photoreceptors since pros plays a role in axon guidance within the embryonic nervous system (Doe et al. 1991; Vaessin et al. 1991). In a wildtype eye, the axons of the outer photoreceptor cells R1R6 synapse onto a retinotopic unit in the first optic ganglion, the lamina (Fig. 1F). The axons of R7 and R8 bypass the lamina and retinotopically project to the second optic ganglion, the medulla. The R7 and R8 axons terminate at two distinct levels in the medulla. To visualize projections of wild-type $\mathrm{R} 7$ axons in the medulla, we filled retinal cells with horseradish peroxidase (HRP). The projections of R1-R6 were densely packed together in the lamina but the individual axons and terminals of R7 were clearly visible in whole mounts of the optic lobe (Fig. 1G). The axons formed optic columns and terminated at evenly spaced intervals to a unique layer of the medulla. HRP eye fills of sev mutant flies confirmed that these axon projections were from R7 cells (Fig. 1H). We filled retinal cells within pros ${ }^{\prime O 1}$ mutant clones and observed a disordered projection pattern (Fig. 1I). R7 axons were irregularly spaced, followed abnormal paths, and terminated at multiple layers in the medulla in a nonretinotopic fashion. The restriction of the phenotype to clones of mutant retinal cells indicates that pros is required in the retina for normal R7 axon connectivity.

pros expression is limited to the $R 7$ equivalence group

The eye phenotype of pros mutants suggests that the gene is involved in determination and differentiation of 

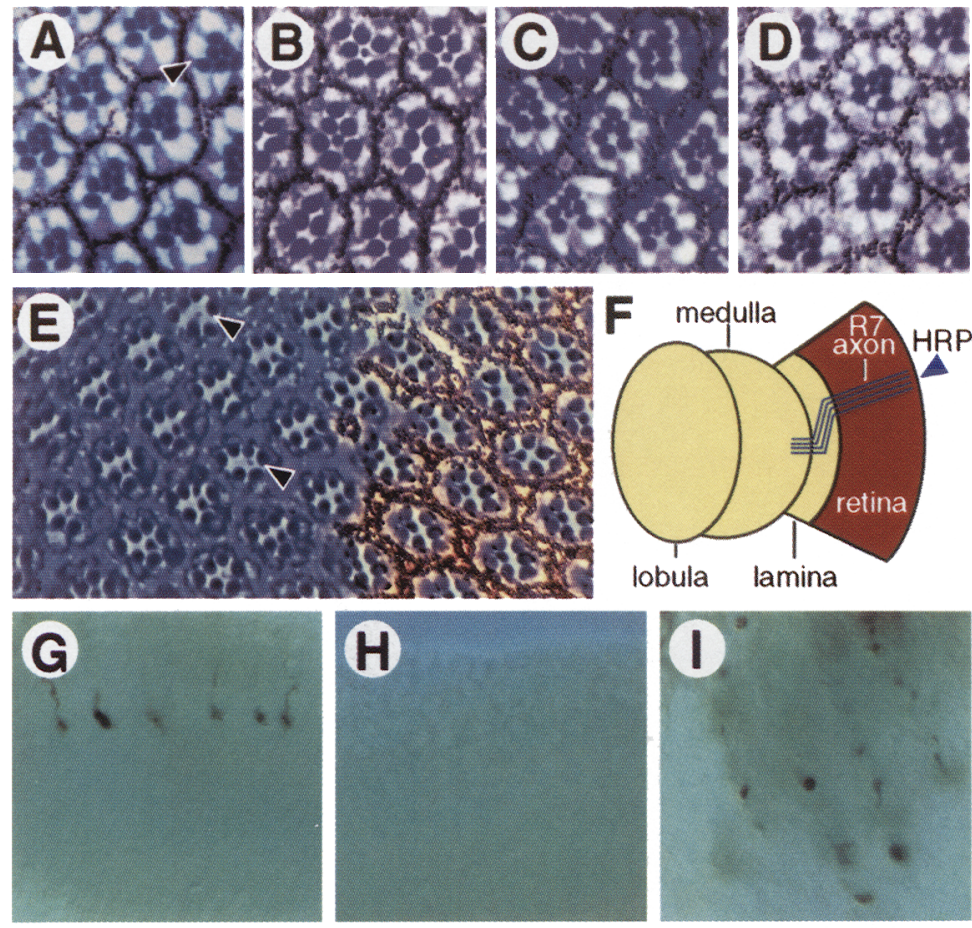

lobula

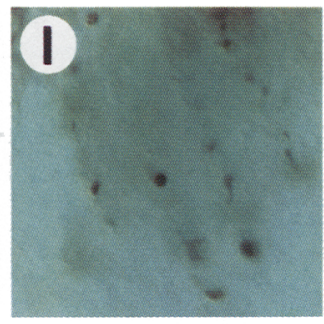

nation of more-basal sections. Therefore, it is not likely that the loss of $\mathrm{R} 7$ and misplacement of 88 cells that accounts for the phenotype. $(F-I)$ pros alters the $\mathrm{R} 7$ retinotopic map. $\{F \mid$ A transverse section through an adult head. The projections of $\mathrm{R} 7$ axons were visualized by application of HRP to particular locations in the retina, which resulted in localized filling of photoreceptors. Superficial and localized application ensures that only a small cluster of neighboring R7 cells (sometime only one) and not R8 cells will be filled (Buckles et al. 1992). (G) Whole mount of a wild-type brain with the retina and lamina removed. A small group of ommatidia wcre filled with HRP, and the terminals and distal axonal segments are seen in this plane of focus. Evenly spaced terminals form a row which corresponds to the terminal zone of the R7 axons. $(H)$ Whole mount of a sevenless brain, as in $G$. The optical plane of focus is at the level of the medulla. No HRP-filled axons were detected, indicating that the filled axons observed in $G$ originate from R7 photoreceptors. (I) Whole mount of brain from a mosaic fly containing a clone of pros eye cells in which photoreceptors within the clone were filled with HRP. Only flies containing large white pros clones (at least 50 ommatidial were used and only flies with fewer than ten filled R7 axons were examined. The mutant axons project together as a group to the appropriate region of the medulla. However they tcrminate at several different levels of the medulla and are not retinotopically ordered in a single terminal zone. The axon paths are grossly normal but meander significantly more than wild-type axons. In some clones virtually all termini were located in different zonal positions. In all clones examined $\leqslant 50 \%$ of termini were positioned in a single layer, suggesting that at least half of the R7 cells connect abnormally. This is significantly more penctrant than the abnormal rhabdomere phenotype of mutant $\mathrm{R} 7$ cells in pros clones.

R7 photoreceptors. To examine the pattern of pros expression in eye imaginal discs, antibodies specific for the pros protein were generated. Antibody staining of eye discs revealed that pros protein is first detected in presumptive R 7 cells of ommatidia $\sim 7-8$ columns posterior to the morphogenetic furrow (Fig. 2A,B). By columns 1112 its expression is also detected in the four cone cells (Fig. 2C). Antibody staining is restricted to the nuclei of these five cells. Significantly, Pros is detected in R7 cells several hours prior to their overt differentiation into neurons, as marked by their expression of the ncural antigen Elav (Fig. 2D). The timing of pros expression in R7 cells suggests that it is induced early in the R7 developmental program. The specificity of the antibody for pros protein was demonstrated by staining eye discs from sev-pros transgenic larvae. In these eye discs, pros should be detected in the cells which express sev: R3, R4, R7, and the four cone cells. The antibody specifically labeled the nu- clei of these cells in sev-pros eye discs (Fig. 2E). Moreover, the antibody labeled cells in the developing nervous system of wild-type embryos in a pattern indistinguishable from the pattern reported previously for pros (Vaessin et al. 1991), whereas the antibody did not label any cells in homozygous mutant pros ${ }^{101}$ embryos /data not shown).

The specific expression of pros in the R7 and cone cell precursors is primarily achieved through transcriptional regulation. We examined the expression pattern of $1 a c Z$ in the enhancer-trap line pros ${ }^{10419}$. The P element in $\operatorname{pros}^{10419}$ is inserted upstream of the transcription start site and expression of the $l a c Z$ reporter gene is restricted to the R7 and cone cells (Fig. 2F, G). Moreover, the onset of $l a c Z$ expression closely resembles the timing of pros expression as determined by antibody staining.

The initial level of pros protein in the presumptive $\mathrm{R} 7$ cell is equivalent to the level seen in the cone cells /cf. 

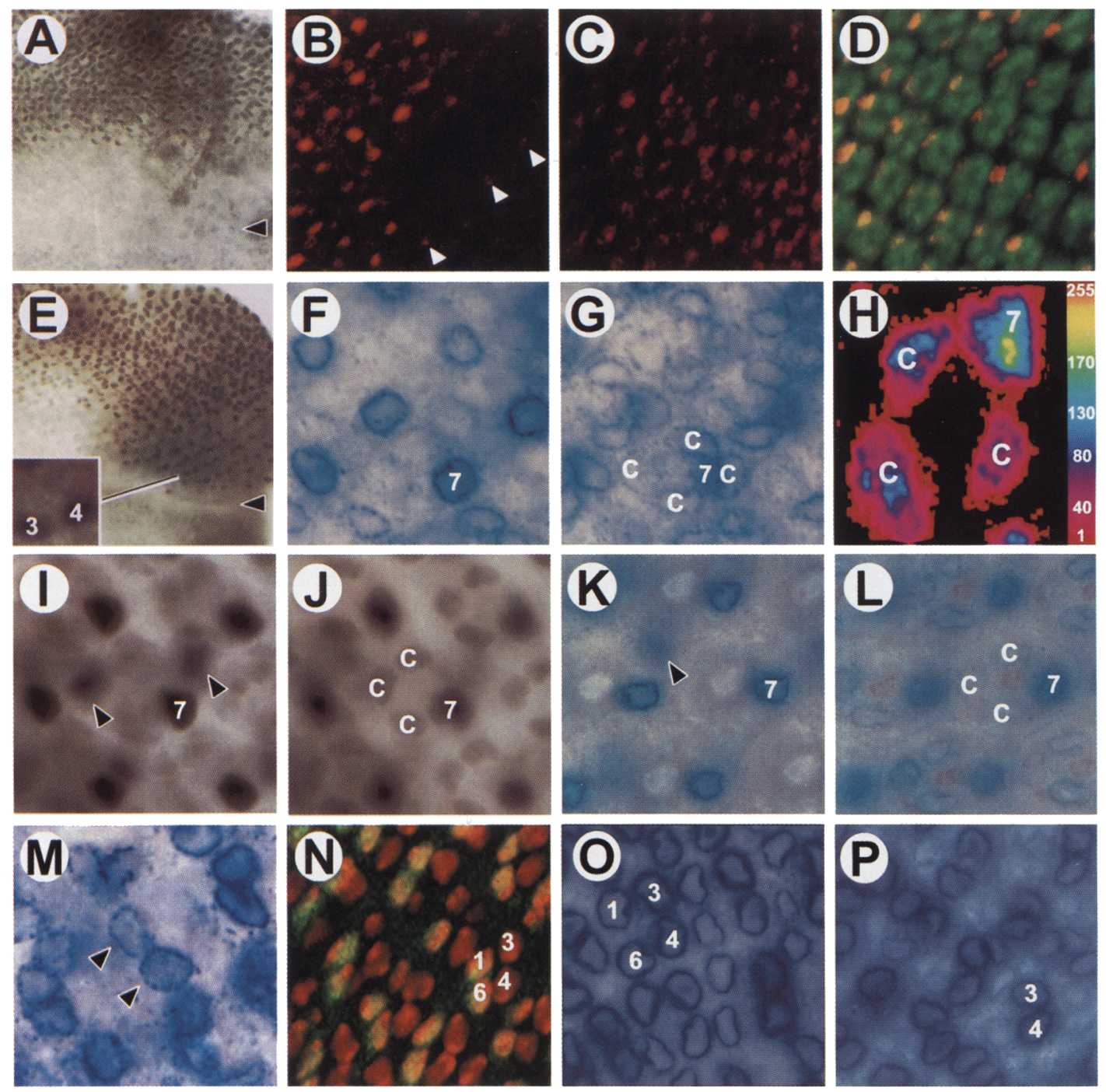

Figure 2. Expression of pros in developing eyes. $(A-E)$ Whole mounts of larval eye discs labeled with antibody against Pros. In $A$ and $E$, discs are oriented with posterior up and are aligned to show similar regions of the disc. $(A)$ Wild-type disc; $(E) P[s e v-p r o s]$ disc. No labeling is observed near the morphogenetic furrow (marked with an arrowhead) in $A$ while staining of clusters of cells in $E$ begins immediately adjacent to the furrow. These cells correspond to R3 and R4, as shown in the insert in $E$. $|B-D|$ are higher magnifications of a wild-type disc labeled with antibody against Pros (shown in red). Anterior is to the right. (B) A basal focal plane showing weak labeling of nuclei from presumptive R7 cells as they are ascending in anterior ommatidia (marked with white arrowheads) and more intense labeling of $R 7$ nuclei in ommatidia beginning three columns posterior. $(C)$ An apical focal plane in a posterior region of the disc showing weak labeling of cone cell nuclei. (D) A disc labeled with antibody against Pros (shown in red) and antibody against Elav (shown in green). Strong staining in both channels appears yellow. The focal plane shows R7 nuclei (red or yellow) and other photoreceptor nuclei (green). (F,G) Larval eye disc from a pros ${ }^{1041 \%} /+$ fly stained with X-Gal. High magnification view of staining of R7 cells (marked 7) at one focal plane $(F)$ and cone cells (marked $c)$ at a more apical focal plane $(G)$. ( $H)$ A disc labeled with pros antibody coupled to FITC was optically sectioned ( $1 \mu \mathrm{m}$ sections) by confocal microscopy. Shown is an apical section of a single ommatidium showing cone cell labeling overlaid with a basal section showing R7 labeling. The signal contribution of the overlying equatorial cone cell was deleted from the composite to keep R7 signal strength constant. Fluorescence intensity was quantitated by NIH Image 6.0 and rendered on a color scale shown to the right. Numbers represent relative intensity values. $(I-L)$ Pupal eye whole mounts $(48 \mathrm{hr}$ after pupation) of wild-type pupae labeled with anti-Pros antibody $(I, I)$ and $\operatorname{pros}^{70419} /+$ pupae stained with X-Gal $(K, L)$. Strong labeling of R7 nuclei with antibody and X-Gal observed at one focal plane $(I, K)$ with labeling of underlying bristle neurons evident (arrowheads). A more-apical focal plane $(J, L)$ shows weak labeling of cone cells. $(M-P)$ Anterior is to the right. $(M)$ Eye disc from a $P\left[s e v-N o t c h{ }^{a c t}\right]$; $\operatorname{pros}^{10419}$ / fly stained with X-gal. Ommatidia viewed at a basal focal plane show two cells (marked with arrowheads) contacting each other in each cluster that stain at a level comparable to a wild-type R7. Apical focal planes not shown here exhibit four weakly staining cells typical of wild-type cone cell staining. No stained cell in the normal R7 position is seen. $(N)$ Confocal image of a $\left.P / s e v-N o t c h{ }^{a c t}\right]$ disc labeled with anti-Pros antibody (green) and with the nuclear dye propidium iodide (red). Staining in both channels appears yellow. The R1, R3, R4, and R6 cells of one ommatidial cluster are labeled. $(O, P) \mathrm{X}$-Gal strongly stains R1, R3, R4, and R6 cells in wild-type flies carrying the H162-lacZ enhancer trap insertion $|O|$, but it stains two cells per ommatidium, R3 and R4, in a P/sev-Notch act background $(P)$. 
cell is equivalent to the level seen in the cone cells (cf. Fig. $2 B, C)$. As the developing ommatidia mature, the level of pros protein in the $\mathrm{R} 7$ cell increases, whereas it remains constant in the cone cells. The increase of pros protein in $\mathrm{R} 7$ cells begins in column 11 which is coincident with the onset of pros expression in the cone cells. In the most mature ommatidia of the larval eye disc, there is a significant difference in levels of pros protein between R7 and cone cells (Fig. 2H). The level of pros protein in the $\mathrm{R} 7$ cell continues to increase as the ommatidia develop during pupal metamorphosis (Fig. 2I,J). pros expression is eventually turned off in all retinal cells between 56-64 hr after pupation (data not shown). The differential expression of pros protein in the eye was mimicked by a similar differential expression of lac $Z$ in the $\operatorname{pros}^{10419}$ enhancer trap line (Fig. 2K,L). This suggests that differential levels of pros protein in $\mathrm{R} 7$ and cone cells is achieved through differential transcriptional activity of pros in R7 and cone cells.

The restriction of pros expression to the $\mathrm{R} 7$ equivalence group is a consequence of the unique time at which these cells are recruited into the ommatidial program. Recruitment of other cells at the same time lcads to their misexpression of pros. We overexpressed an activated form of the Notch receptor in defined cells, which transiently blocks their proper recruitment and causes them to adopt different fates (Fortini et al. 1993). In eye discs from flies carrying the $s e v-N^{a c t}$ gene, we observed two additional cells within each ommatidium that expressed pros at levels comparable to wild-type R7 cells (Fig. 2M). This result is consistent with the observation that two outer photoreceptor cells are developmentally delayed and adopt an R7 cell fate (Fortini et al. 1993). It suggests that any cell that is competent to receive a specific cue at this time in development can express pros. Interestingly, this period of competence appears to be fairly extended because developmental delay of the cone cell precursors by $\operatorname{sev}-N^{a c t}$ has no effect on their expression of pros idata not shown).

To determine the identities of the two ectopically stained cells, we used propidium iodide to label all of the nuclei in the eye disc and double-labeled with the Pros antibody. At the eight-cell cluster stage when the nuclei of R3, R4, R1, and R6 are at the apical surface, the Pros antibody labeled the two cells in the posterior position that by position correspond to R1 and R6 (Fig. 2N). The $\mathrm{R} 1$ and R6 precursor cells, developmentally delayed by sev- $N^{a c t}$, appear to be recruited into the R7 equivalence group and express pros. To verify that the R1 and R6 precursor cells were recruited into the $\mathrm{R} 7$ equivalence group, we examined the effect of $s e v-N^{o c t}$ on seven-up $(s v p)$ gene expression. $s v p$ is expressed in the outer photoreceptor cells R1, R3, R4, and R6 (Mlodzik et al. 1990; Fig. 2O). The addition of sev- $N^{a c t}$ resulted in the loss of svp expression in the R1 and R6 cells (Fig. 2P).

\section{Regulation of pros expression by the Sev-Ras1 pathway}

The R7 and cone cell precursors have an equivalent developmental potential that is restricted by Sev RTK ac- tivity. This suggested the possibility that sev might induce pros expression to higher levels in developing R7 cells. We compared the pros expression pattern in wild type and sev mutant eye discs (Fig. 3A,B). Cone cells in null-mutant sev discs expressed pros at a level comparable to wild type. However, no elevated level of pros expression was observed in the transformed R7 cells. Conversely, an average of three cells per ommatidium expressed elevated levels of pros in a constitutively activated $\operatorname{sev}$ mutant, $\operatorname{SeV}^{\mathrm{S} 11}$ (Fig. 3C). The positions of these cells suggest they are the endogenous R7 cell and cone cells transformed to an $\mathrm{R} 7$ fate. These results indicate that Sev signaling is not required for normal pros expression in cone cells, nor is it required for low level expression in the R7 cell. However, it is necessary and sufficient for upregulated expression of pros.

Sev signaling is mediated by Ras1. To determine the role of Ras 1 in pros expression, a constitutively activated Ras1 mutant, sev-Ras1 ${ }^{v 12}$, was examined (Fig. 3D). Like $\mathrm{Sev}^{\mathrm{S11}}$, this mutant exhibited elevated pros expression in the endogenous R7 cell and the cone cells that were transformed to an $\mathrm{R} 7$ fate. This was observed even in a
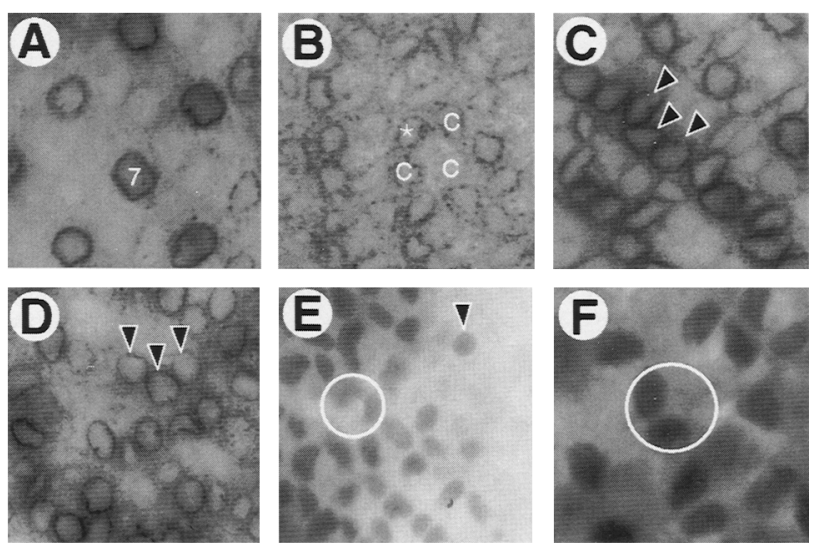

Figure 3. Regulation of pros expression by the Sev-Ras1 pathway. $(A-D) \mathrm{X}$-Gal staining of pros $^{1041 \%} /+$ eye discs. All panels are oriented with anterior to the right. $(A)$ Wild-type disc showing $\mathrm{R} 7$ cell staining; $(B) \operatorname{sev}^{d 2}$ eye disc. Note that the transformed R7 cell staining $(*)$ is equivalent to cone cell staining (marked c). (C) Sev ${ }^{S 11}$ disc; (D) P[sev-Ras1 $\left.{ }^{\text {v12 }}\right]$ disc. Arrowheads point to cells within one ommatidium that intensely stain with $\mathrm{X}$-Gal. $(E, F)$ Whole mounts of $P\left[\mathrm{seV}-\mathrm{Ras} 1^{\mathrm{N} 17}\right.$ ) eye discs labeled with anti-Pros antibody. The discs have been overstained to more readily visualize the weak labeling of cone cell nuclei. The region of the disc in $E$ is $\sim 7-10$ rows behind the furrow, where Pros-labeled cells normally first appear. An R7 cell (arrowhead) is labeled at the same intensity as the cone cells. A reduced number of R7 precursor cells is labeled in this region of the disc. More posterior in $E$, a circle outlines a typical ommatidium containing two labeled cells that by position and morphology are indicative of cone cells. There is no evidence of intense R7 cell staining at this stage of development. The region of the disc in $F$ is near the posterior edge where normally four cone cells are labeled. The circle in $F$ highlights a typical ommatidium with two labeled cells that lie in the apical focal plane typical for cone cell nuclei. 
sev null background (data not shown). Thus, Ras1 is genetically downstream of $\mathrm{sev}$ in regulating pros expression. To examine the effects of removing Rasl function in retinal cells, a dominant negative form of Ras1 (sev$R a s 1^{N 17}$ ) was used. The eye phenotype of $\mathrm{seV}-\mathrm{Ras} 1^{\mathrm{N1} 7}$ is the absence of R7 cells, presumably because of their transformation to a cone cell fate (Allard et al. 1996). We examined pros expression in sev-Ras $1^{N 17}$ mutant eye discs and found that $\sim 50 \%$ of the cone and $\mathrm{R} 7$ cells did not express pros, nor did they express elevated levels of pros typical of an R7 cell (Fig. 3E,F). Therefore Ras1, like sev, appears to be both necessary and sufficient to activate high-level expression. However Ras1, unlike sev, is also required for low-level expression in both R7 and cone cell precursors.

\section{Regulation of pros expression by MAPK-responsive transcription factors}

To further define the role of the Rasl signaling pathway on pros expression, we examined the effects of mutations in two genes encoding ETS-transcription factors: yan and pnt. The activities of both factors are regulated by MAPK phosphorylation and both factors are critical for R7 fate determination (Brunner et al. 1994a; O'Neill et al. 1994; Rebay and Rubin 1995). We tested pros for dominant genetic interactions with partial loss-of-function alleles of yan and pnt. In flies carrying the viable allelic combination $p n t^{\Delta 88} / p n t^{T c}, 25 \%(n=820)$ of the ommatidia are missing $\mathrm{R} 7$ cells (data not shown). The $p n t^{\Delta 88} / p n t^{T C}$ phenotype was dominantly enhanced by $\operatorname{pros}^{\prime 01}$, leading to a roughening of the external eye (Fig. $4 \mathrm{~A}, \mathrm{~B})$. This was accompanied by a reduction in $\mathrm{R} 7$ cells from $\sim 0.75( \pm 0.03$ S.D.) cells per ommatidium to $\sim 0.55$ $( \pm 0.04$ S.D. $)$ cells per ommatidium $(n=1690)$. Conversely, the yan ${ }^{1}$ rough eye phenotype was dominantly suppressed by pros $^{101}$ (Fig. 4C,D) and was accompanied by a reduction in supernumerary $\mathrm{R} 7$ cells from $1.8( \pm 0.2)$ cells per ommatidium to $1.3( \pm 0.1)$ cells per ommatidium $(\mathrm{n}=1670)$. The effect of pros ${ }^{101}$ on these phenotypes was partial, suggesting that pros is not a major limiting component when yan and pnt activities are reduced.

To examine the effects of yan and pnt on pros expression, we stained mutant eye discs with anti-Pros antibodies. Partial loss-of-function $p n t^{\Delta 88} / p n t^{T 6}$ mutants contained a markedly reduced number of pros-expressing cells (Fig. 4E,F). Only two or three pros-positive cone cells per ommatidium were observed, and no elevated expression in R7 cells was observed. Thus, pnt is required for pros expression in both the cone cell and R7 cell precursors. In contrast, the partial loss-of-function yan ${ }^{1}$ mutant contained a number of cells of unknown identity that ectopically expressed pros in addition to the presumptive R7 and cone cells (Fig. 4G). The positions of the cells suggest they are recruited from the uncommitted cell population. This result is consistent with the ectopic transformation of uncommitted cells to an R7 fate observed in a yan ${ }^{1}$ mutant (Lai and Rubin 1992), and suggests that Yan normally represses pros expression.

Yan is hypothesized to be a repressor of gene transcrip-
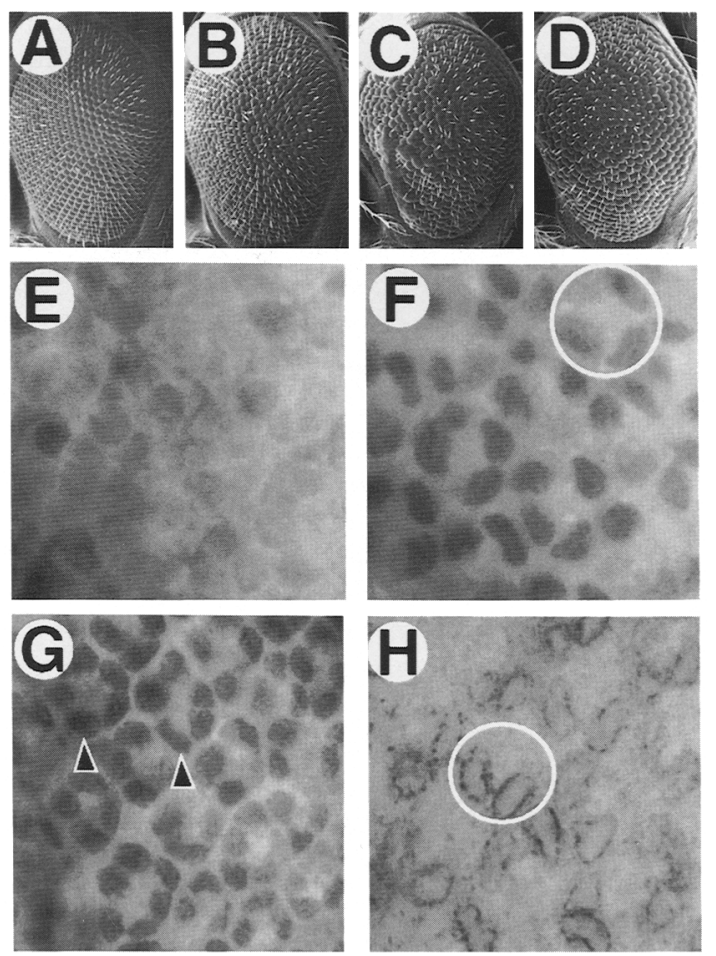

Figure 4. Regulation of Pros expression by two MAPK-responsive factors. $(A-D)$ Scanning electron micrographs of the com-

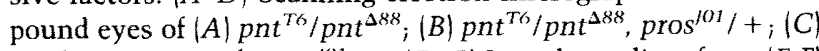
yan ${ }^{t}$; and $|D|$ yan $^{t} ;$ pros $^{\prime \prime 1} /+.(E-G)$ Larval eye discs from $|E, F|$ $p^{T} t^{T 6 / p n t} t^{\Delta \& 8}$ and $(G)$ yan $^{1}$ flies labeled with anti-Pros antibody. Anterior is to the right. $(E)$ pnt mutant discs are often missing the strongly labeled nuclei that correspond to R7 nuclei in wild type. $(F)$ pnt mutant ommatidia have an average of three labeled nuclei (an example is circled) which lie in an apical focal plane typical for cone cell nuclei. $(G)$ Ommatidia in yan mutant discs typically contain six and occasionally seven labeled cells at different focal planes. This apical focal plane shows several ommatidia with five labeled cells. Two of these ommatidia are indicated with arrowheads. Note the variable labeling intensity between cells. Often three or more cells are strongly labeled with the antibody. $(H) P\left[s e v-y a n^{A c t}\right]$; $\operatorname{pros}^{1041 \%} /+$ eye disc stained with X-gal shows an average of two labeled cells per ommatidium. A typical ommatidium is highlighted with a circle. The apical positions of the nuclei and their morphology indicate that they are cone cells. The intensity of staining is approximately equivalent between cells with no consistent single cell staining more strongly than the others.

tion that is down-regulated when phosphorylated by MAPK (Rebay and Rubin 1995). If pros transcription is normally activated by Yan phosphorylation, then unphosphorylated Yan should constitutively repress pros transcription. A dominant activated form of yan $\left(\mathrm{yan}^{A C T}\right)$, in which all potential MAPK phosphorylation sites are missing, blocks cell differentiation in the eye and constitutively represses transcription of reporter genes in cell culture (Rebay and Rubin 1995). We examined pros expression in flies carrying the $\operatorname{yan}^{A C T}$ gene under control of the sev enhancer $\left(\mathrm{sev-yan}{ }^{A C T}\right)$. Most ommatidia contained substantially fewer cone and R7 
cells that expressed pros, and elevated pros expression was not observed in R7 cells (Fig. 4H). This suggests that activation of pros transcription in all cells within the $\mathrm{R} 7$ equivalence group requires the down-regulation of Yan activity through phosphorylation by MAPK in the R7 and cone cell precursors. This is also consistent with the established role for Rasl in activating pros expression.

The suppression of pros expression by $\mathrm{sev}-\mathrm{Ras} 1^{\mathrm{N17}}$, $p n t^{\Delta 88} / p^{2} t^{T 6}$, and sev-yan ${ }^{A C T}$ mutants was not complete, and indicates that these alleles may contain some residual activity. Although this is very likely for the $p n t^{\Delta 88} /$ pnt $^{T 6}$ mutant, it is not as clear for the other two genes. It is possible that the signal needed to initiate the low level of pros expression in the equivalence group can occur before the sev enhancer becomes active, and, therefore, before yan ${ }^{\mathrm{ACT}}$ and Ras $1^{\mathrm{N} 17}$ are synthesized in these cells.

\section{Activation of pros expression by a parallel pathway}

The experiments described above suggest that Yan and Pnt may act on pros transcription in response to Ras1 activity. It was unclear whether their activities are sufficient for Ras 1 signaling, because nuclear factors such as Sina and Phyl are also required for R7 determination. Therefore, we examined various sina and phyl mutants to see whether these genes are required for normal pros expression. A sina null mutant expressed levels of pros in the R7 equivalence group comparable to wild typc, with elevated levels in transformed R7 cells equivalent to or sometimes greater than that observed in wild-type R7 cells (Fig. 5A,B). Because sina is absolutely required for $\mathrm{R} 7$ determination, this result suggests that elevated levels of pros expression are not strictly coupled to R7 fate determination. It also contrasts with the observed absence of elevated expression in the transformed cells of a sev null mutant. We examined pros expression in $\mathrm{sev}$ sina double mutant eye discs to determine the relationship between sev and sina in regulating pros transcription. Expression in the double mutant resembled expression in the sev mutant (Fig. 5C), indicating that sev is epistatic to sina. Consistent with this result, the elevated expression of pros in transformed cone cells in a $\mathrm{SeV}^{\mathrm{S11}}$ mutant was not significantly affected by sina null mutations (data not shown). These epistasis results suggest that $s e v$ acts downstream or in parallel to sina.

We also examined the effect of removing phyl function during R7 development. The transformed R7 cells in a phyl mutant expressed pros at a level comparable to wild type (Fig. 5D). This result suggests that phyl is not absolutely required for elevated pros expression. However, the presence of phyl in cells is sufficient to induce them to express elevated levels of pros. We examined eye discs from flies bearing the phyl gene under control of the sev enhancer (2sev-phyl). Ectopic expression of phyl in the cone cell precursors resulted in supernumerary cells that express pros at levels comparable to the R7 cell (Fig. 5E). These cells appeared by position to correspond to transformed cone cells. We conclude that expression of phyl in cone cells is sufficient to induce elevated levels of pros
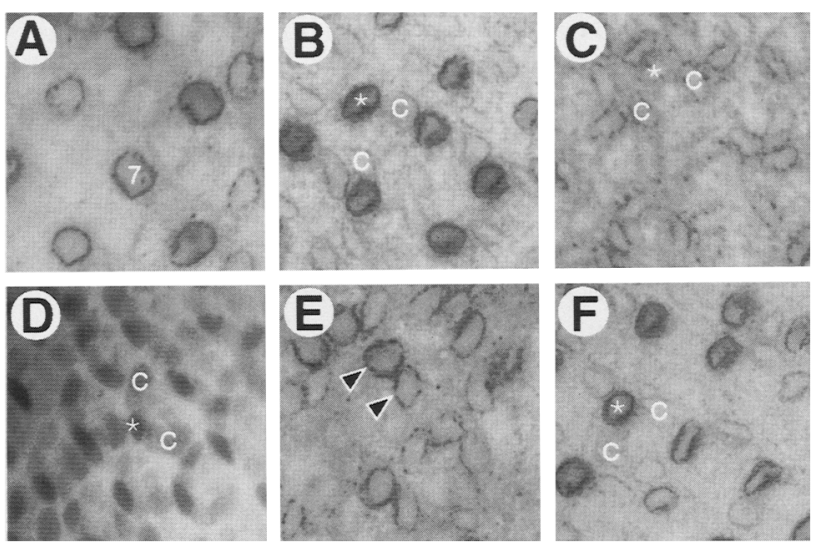

Figure 5. Sina and Phyl activate pros expression. X-gal staining of $\operatorname{pros}^{1041 \%} /+$ eye discs $(A-C, E, F)$ or labeling with antibody against Pros $(D)$. All panels are oriented with anterior right. $(A)$ wild-type disc showing $\mathrm{R} 7$ cell staining; $(B) \sin a^{2}$ eye disc. Staining of cone cells (marked $\mathrm{c}$ ) is equivalent to wild type, and staining of transformed R7 cells (one is marked with *) is somewhat stronger than wild type. $(C) \mathrm{sev}^{\mathrm{dz}}$; sina eye disc. Intense staining of transformed $\mathrm{R} 7$ cell is abolished and weak cone cell staining appears unaffected. $(D) p h y l^{2} / p h y l^{4}$ disc. An average of one cell per ommatidium is intensely stained $(*)$, which by position corresponds to a transformed R7 cell. Posterior in the disc, the ommatidial cell clusters become disorganized and up to five cells display weak staining (data not shown). Because R1/R6 adopt a cone ccll fate in phyl mutants, these ectopically expressing cells may be transformed R1/R6 cells. (E) P/2sevphyl/ eye disc. Multiple cells per ommatidium are staining intensely with X-Gal. The position of these cells correspond to transformed cone cells. They all lie on the same focal plane in a position characteristic of $\mathrm{R} 7$ cells in this region of the disc. Two strongly staining cells within one ommatidium are marked with arrowheads. The increased number of highly expressing cells was accompanied by a decrease in the number of cells expressing lower levels of pros normally observed in cone cells. The transformation of cone cell identity in $2 \mathrm{sev}-$ phyl mutants results in fewer cone cells per ommatidium (Dickson et al. 1995 ) which likely accounts for the loss of lower-expressing cells. $(F) P[2 s e v-p h y l]$ sina $a^{2}$ eye disc. One cell per ommatidium which occupies the position of the transformed R7 stains strongly with X-Gal $(*)$. The cone cells stain comparably to wild type.

expression. Possibly, one reason that phyl is not absolutely required for pros upregulation in the $\mathrm{R} 7 \mathrm{cell}$ is that its function is redundant; other factors compensate for its absence.

Increased expression of pros in transformed 2sev-phyl cells required the sina gene. No supernumerary cells with elevated levels of pros were observed in eye discs from 2sev-phyl sina flies (Fig. 5F). This result suggests that phyl requires sina activity to stimulate pros expression.

\section{Sina protein forms a complex with Phyl}

The close genetic interaction between sina and phyl suggested that the proteins may physically associate and 
that this association may play an important role in regulating pros expression. To test this, we employed the yeast two-hybrid system that detects protein interactions (Gyuris et al. 1993). Full-length Sina protein was fused to a transcriptional transactivation domain (SinaTA) and Phyl protein was fused to a LexA DNA-binding domain (Phyl-LexA). Interaction was observed by both proteins together transactivating a lacZ reporter gene (Fig. 6A). In addition, both proteins together transactivated a Leucine reporter gene, resulting in transformant growth on Leu - media (data not shown). Comparable transactivation was observed when the fusion domains were swapped, using Sina-LexA and Phyl-TA. Deletion of amino acids $108-400$ of Phyl $(\Delta 108)$ abolished the interaction, whereas deletion of amino acids $131-400$ $(\Delta 131)$ resulted in a strong interaction, as measured by lac $Z$ transactivation. This suggests that amino acids between positions 108 and 130 are critical for interaction. The strength of the $\Delta 131$ interaction is 27 -fold greater than the interaction with full-length Phyl. This difference may reflect a peculiarity of one of the constructs or may be of biological relevance. The interaction between Sina and Phyl is specific since bicoid fused to LexA only

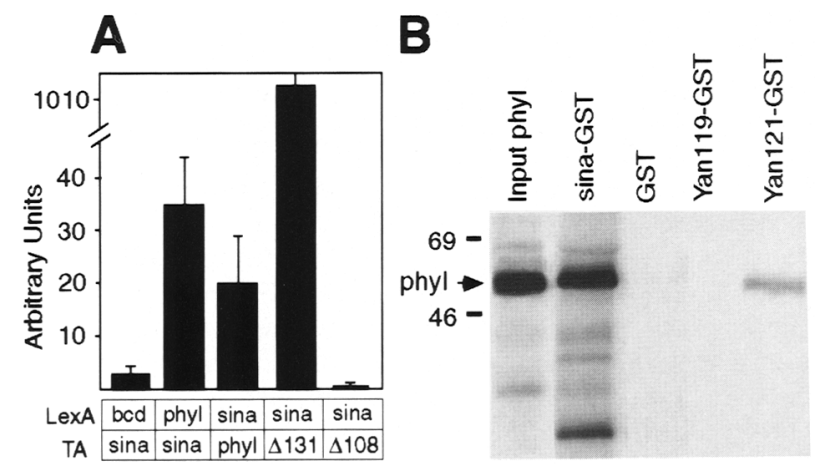

Figure 6. Interaction of Sina and Phyl proteins. $|A|$ Quantitative assays of $\beta$-galactosidase activity from the $1 a c Z$ reporter gene in various yeast transformants. Exponential-phase yeast containing the indicated fusion proteins were assayed for $\beta$-galactosidase activity with ONPG substrate. LexA-Sina and LexA-Phyl are full-length fusion proteins; LexA-Bcd, a negative control fusion, expresses amino acids 2-160 of Bicoid (Gyuris et al. 1993); TA-Sina and TA-Phyl are full length fusions to an acidic activation domain (TA); TA- 131 and TA$\Delta 108$ are fusions of the TA domain to Phyl with amino acids $131-400$ and 108-400 deleted, respectively. Activity was calculated as the difference in specific activity (Ausubel et al. 1993) between each transformant grown in galactose (induces TAfusion protein synthesis) and each grown in glucose (TA-fusion synthesis uninduced). Values presented are the average of duplicate assays of 4-20 independent transformants. Error bars represent standard deviation. The error bar for Sina/Phyl $\Delta 131$ is \pm 75 . (B) $\left[{ }^{35} \mathrm{~S}\right]$ methionine-labeled, in vitro translation of Phyl analyzed on a SDS-polyacrylamide gel. The protein was either electrophoresed directly following translation or following glutathione-sepharose adsorption in the presence of Sina-GST, GST, Yan119-GST, or Yan121-GST. Ten percent total phyl protein input was loaded into lane 1 . The positions of molecular weight markers are given. weakly transactivated $l a c Z$ in the presence of Sina-TA. To confirm the apparent in vivo association of Sina and Phyl, in vitro association assays were performed (Fig. 6B). Phyl protein was translated in vitro and incubated with a Sina-glutathione S-transferase (Sina-GST) fusion protein immobilized to glutathione beads. Phyl remained bound to immobilized Sina-GST following extensive washing of the beads, whereas it was not bound to immobilized Yan-GST or GST alone. Moreover, in control experiments Sina-GST did not associate with heterologous in vitro translated proteins such as TAF60, TAF30 $\alpha$, and TFIIE $\alpha$ (data not shown). These results establish that Phyl interacts specifically with Sina.

\section{Discussion}

\section{The role of pros in photoreceptor development}

We have found that pros is required for proper $\mathrm{R} 7$ differentiation and axon connectivity in the adult retina. It is not absolutely required for $\mathrm{R} 7$ fate determination because loss of pros function causes no significant transformation between different cell types. However, pros may participate in $\mathrm{R} 7$ determination because it genetically interacts with sina, pnt, and yan in forming $\mathbf{R} 7$ cells. pros appears to control neural connectivity indirectly by regulating gene expression because the protein is nuclear localized and contains a divergent homeo domain. Although pros is expressed in the cone cells at a low level, it is unclear if it is needed for their differentiation.

\section{Regulation of pros expression}

The expression of pros is initially activated in the five cells that comprise the R7 equivalence group of the eye disc. Later, R7 cells exhibit elevated levels of pros expression as a response to Sev-mediated activation while transcription remains constant in the cone cells. Several lines of evidence suggest that regulation occurs by a twostep process. First, pros expression is initially detected in the R7 precursor cells prior to the estimated point of time when sev is activated (Mullins and Rubin 1991). Second, initial detection of pros expression in the $\mathrm{R} 7$ precursor cell of sev mutants occurs at the same time as in wild type. Finally, cone cell precursors transformed with activated sev exhibit an initial low level of expression followed by a high level of expression.

It is possible that there is a causal relationship between the initial phase of pros transcription and the subsequent ability to be induced by Sev. Mutations in Ras1 and yan which block the first step also block the second step. It would imply that some genes such as pros are able to respond to induction only by having independently established transcriptional activity. This has been observed with $\mathrm{MyoD}$ activation during mesoderm induction in Xenopus (Rupp and Weintraub 1991). Thus, competence of cells to respond to inductive signals could be regulated by the transcriptional responsiveness of genes. These genes might be required for determination or dif- 
ferentiation. Some genes such as phyl may be regulated not by a two-step mechanism but by direct activation. Perhaps a single inductive interaction involves a combination of different modes of transcriptional activation. Our results suggest that after initiation of pros transcription, Sev-mediated induction leads to the accumulation of pros protein above a threshold level in the R7 cell. This level of pros protein might establish a positive feedback loop that stabilizes pros expression into pupal development, long after Sev signaling is complete. In cone cells, the low level of pros protein might lead to stable but low-level expression.

\section{Transcription in the $R 7$ equivalence group}

Here, we report evidence implicating Ras 1 signaling in the regulation of gene transcription in the $R 7$ equivalence group. This is based on three findings that were made for pros transcription. First, a dominant negative form of Ras 1 can block pros expression in any cell of the equivalence group. Second, a constitutively repressive form of yan that is unable to be phosphorylated by MAPK can block pros expression in the equivalence group. Third, in the absence of pnt activity, cells in the equivalence group fail to express pros. The first result establishes that Ras1 activity is required for pros transcription. The second result argues that Ras1 activates pros transcription by downregulating yan activity through a modulation of MAPK activity. The third result argues that pnt activation is critical for pros transcription.

One simple hypothesis to account for the roles of these factors is that the basal level of yan activity is sufficient to repress pros transcription and the basal level of pnt activity is unable to activate pros. Activation of Ras 1 in cells of the R7 equivalence group counteracts the repression of pros by yan (by stimulating MAPK to phosphorylate Yan) and activates pnt to regulate positively pros transcription (Fig. 7A). This model also explains our observation that the absence of yan causes pros expression in supernumerary cells. Several additional points about this model are worth noting. First, the nature of the signal that activates Rasl in the R7 equivalence group is unknown. Possibly, the signal is mediated by contacts between uncommitted cells and differentiating photoreceptors in accordance with the sequential inductive mechanism operating at earlier stages of eye development (Zipursky and Rubin 1994). Formation of extra photoreceptor cells leads to formation of extra cone cells as a secondary event (Basler et al. 1991), possibly by increasing the number of cell contacts between differentiated and uncommitted cells. Second, activation of pros transcription depends on developmental timing. Rasl is activated in all photoreceptor cells and yet only cells in the R7 equivalence group express pros. However, transient delay of R1 and R6 precursor cell fate determination leads to these cells expressing pros. We suggest that a signal timed to occur after R1 and R6 determination confers a specific transcriptional response within cells competent to receive the signal. This competence could

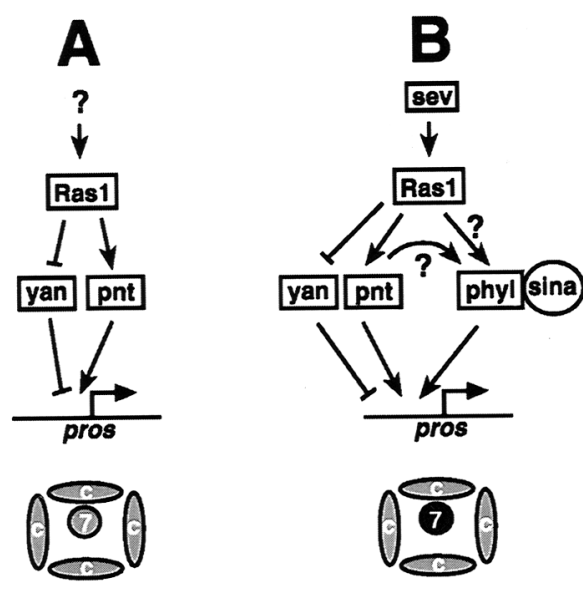

Figure 7. Model for the regulation of pros expression in the equivalence group. $(A)$ Activation of Rasl by an unknown mechanism results in the transcription of pros. Although our experiments do not distinguish whether Yan and Pnt act upstream or downstream from Rasl, we favor the view that they act downstream because of their demonstrated positions downstream of Ras1 in R7 determination. We propose that Ras1 acts through MAPK to alter the activities of the transcription factors Yan and Pnt. They, in turn, may bind to the ETS DNA binding sites upstream of pros to directly regulate pros transcription. $(B)$ Within the presumptive R7 cell, Sev RTK is activated and signals through Ras 1 to upregulate pros expression. Activation via Sev may result in a stronger or more sustained response of MAPK activity and modulate Yan and Pnt activity to a greater degree. The presumptive R7 cell also contains Phyl and Sina which interact, and together they also upregulate pros transcription. It is possible that synthesis of Phyl in the presumptive R7 cell is caused by activation of Sev and Rasl, with the participation of Yan and Pnt.

be manifested through use of alternative signaling pathways not normally utilized in the other photoreceptors or by inhibition of the signal response in cells already undergoing differentiation. Third, our experiments do not address the possibility that Yan and Pnt act antagonistically toward each other by direct competition for common binding sites in the pros gene. However, both purified Yan and Pnt proteins bind specifically to two 5 '-flanking sequences upstream of the pros transcription start site (R.C. Kauffmann and J. Zhang, unpubl.). Possibly, Yan binds to regulatory sequences with higher affinity than Pnt, occupying these elements and occluding Pnt from the gene.

\section{Transcription in the $R 7$ cell}

Expression of pros is upregulated in cells of the equivalence group by Sev induction. In Figure $7 \mathrm{~B}$, we outline a hypothetical pathway for the relationship between $\mathrm{sev}$ and pros. A critical intermediate is Ras1; constitutively activated Ras1 is sufficient to up-regulate pros transcription, while a dominant negative form of Ras 1 blocks sev activation of pros. Likewise, yan and pnt are likely intermediates in sev signal transduction because absence 
of pnt activity or constitutive yan activity blocks sevmediated upregulation of pros.

The placement of phyl and sina into the pathway is more ambiguous. However, we propose that sina and phyl function in parallel to another pathway downstream of Ras1. There are two reasons for our proposal. First, only R7 cells that have activated Sev normally upregulate pros. However, when phyl is ectopically synthesized in cone cells, pros is up-regulated in a sev-independent manner. This up-regulation depends on sina activity. Second, the absence of phyl or sina in an R7 cell has no effect on sev-dependent upregulation of pros. The first result argues that phyl activity is sufficient to up-regulate pros transcription provided sina is also present. The second result suggests that sina and phyl do not up-regulate pros transcription singlehandedly in an R7 cell. A second pathway makes their functions redundant. Possibly, the second pathway utilizes yan and pnt, which may be modulated to a greater degree in R7 than in other equivalence group members. In support of this hypothesis, it has been observed that Yan stability is lower in R7 than in cone cells (Rebay and Rubin 1995).

A simple hypothesis to account for placement of $\sin a$ and phyl in a common pathway is that the two proteins must directly interact to be completely active. In this model, either protein by itself is inactive, but when complexed together they function synergistically to regulate transcription. Complex formation would normally be restricted to the R1/R6 and R7 cell subtypes, ensuring that synergistic activation is limited to these cells. Such a mechanism is reminiscent of muscle-specific gene expression that requires both ubiquitous and myogenic bHLH factors (Lassar et al. 1991). This model accounts for our finding that Sina and Phyl proteins interact in vivo and in vitro. An alternative model that also fits our data is that interaction between Phyl and Sina is transient, resulting in activation of Sina.

\section{Concluding remarks}

Ras 1 signaling is required to regulate pros expression at low levels in the equivalence group and at high levels in R7. How does signaling through the Ras l pathway result in two different responses? In PCl2 cells, treatment with NGF leads to neural differentiation, whereas treatment with EGF leads to cell proliferation (Marshall 1995). Both factors signal through Ras and MAPK activation. The addition of NGF results in an elevation of RasGTP levels and prolonged MAPK activation for several hours. In contrast, increased levels of RasGTP and activation of MAPK persist for only a short time following stimulation by EGF. Likewise, the signal that activates Ras 1 in the R7 equivalence group may lead only to transient activation of Rasl and, by inference, MAPK. Transient activation of the signaling pathway would result in modest activation of the pros gene. Conversely, activation of the Sev RTK may result in prolonged activation of Ras 1 and MAPK to generate higher levels of transcription of pros and initiate transcription of other target genes.

\section{Materials and methods}

Genetics

The following fly strains were used for genetic interactions: sev-N $N^{\text {Act }}$ (Fortini et al. 1993); sev-Ras ${ }^{\text {v12 }}$ (Fortini et al. 1992); sev-Ras ${ }^{N 17}$ (Allard et al. 1996); yan ${ }^{1}$ (Lai and Rubin 1992); sevYan $^{\text {Act }}$ (Rebay and Rubin 1995); 2sev-phyl, phyl ${ }^{2}$, and phyl ${ }^{4}$ (Dickson et al. 1995); $\operatorname{Sev}^{S 11}$ (Basler et al. 1991); pnt ${ }^{\Delta 88}$ and $p n t^{T 6}$ (Brunner et al. 1994a). The recessive lethal P-element insertion line 1/3)10419 or pros $^{10419}$ was obtained from the Spradling enhancer trap stock collection. The cytological map position of 1(3)10419 was determined to be $86 \mathrm{E}$ (Berkeley Drosophila Genome Project). Complementation analysis of $1(3) 10419$ and pros $^{\prime 01}$ indicated they were allelic. The $1(3) 10419$ P-element in serted $5^{\prime}$ of the gene in the promoter region as determined by Southern analysis. Clonal analysis in the eye was performed on pros $^{\prime 01}$ using the FLP/FRT system (Xu and Rubin 1993).

The $P$ /sev-pros/ transformation vector was made by cloning a 5.3-kb pros cDNA (Vaessin et al. 1991) into a sev expression plasmid (Chang et al. 1995). Transgenic lines were generated by p-element-mediated germ-line transformation.

\section{Prospero antibodies}

The start codon of pros cDNA was replaced with a DNA sequence encoding $\mathrm{MetHis}_{6} \mathrm{Gly}_{2}$ by PCR mediated site-directed mutagenesis. An NdeI-BamHI fragment from the engineered DNA corresponding to Met-1 to Ser-408 /Vaessin et al. 1991; GenBank accession number M81389) of the Pros protein was inserted into $\mathrm{pET} 3 \mathrm{c}$ to generate pProsl. The Prosl protein was purified from bacterial lysate by $\mathrm{Ni}^{2+}-\mathrm{NTA}$ Agarose (Qiagen) affinity column and subsequently preparative SDS-PAGE for the immunization of mice (Harlow and Lane 1988). Affinity purified antibodies were prepared by passing serum over a Pros 1 affinity column and eluting with $0.1 \mathrm{M}$ glycine- $\mathrm{HCl}(\mathrm{pH} 2.5)$.

\section{Histology and immunohistochemistry}

Eye fills were done as previously described (Buckles et al. 1992). The adult heads were fixed and embedded, and sections were cut as described by Carthew and Rubin (1990). Samples were prepared for scanning electron microscopy as described by Kimmel et al. (1990) with the exception that hexamethyldisilazane (Ted Pella) was substituted for freon. The $\beta$-galactosidase activity of pros ${ }^{10419}$ discs was assayed with X-gal as described by Moses and Rubin (1991).

Immunolabeling of eye discs and pupal retinas by was done as described by Zheng et al. (1995). For immunofluorescence labeling, Cy3- and FITC-conjugated IgGs were used (Chemicon and Cappel, respectively). Staining of nuclei with propidium iodide was done as described in Neufield and Rubin (1994).

\section{Expression plasmids}

Plasmids expressing Yan119-GST (amino-terminal region of Yan with amino acids 1-389) and Yan 121-GST (carboxy-terminal region of Yan with amino acids 388-732) were provided by Z.C. Lai (Pennsylvania State University, State College). pP$\mathrm{ntC} / \mathrm{GST}$ was generated by ligating a $0.6-\mathrm{kb}$ Sacl-Sall fragment from pACpntP2 (O'Neill et al. 1994) into pGex-3X to generate $\mathrm{pPntC/GST}$. The carboxy-terminal region of PntP2 from Ser479 to Asp-718 is linked to GST through a single serine linker. pSina/GST was constructed by PCR amplification of Sina sequences from position 903 to 1851 (Carthew and Rubin 1990) and insertion into pGex-2T to create pSina/GST. phyl cDNA sequence was amplified by PCR from nucleotides 234 
1446 (Chang et al. 1995) and was inserted into pET3c to create pET/Phyl.

The Phyl two-hybrid expression plasmids were derived from the vectors pEG202 and pIG4-5 (Gyuris et al. 1993). Phyl PCR product $(234-1446)$ was inserted into pJG4-5 and pEG202 to generate pIG4-5/Phyl and pEG202/Phyl, respectively. Delction mutants Phyl $\Delta 130-400$ and Phyl $\Delta 107-400$ were constructed by a partial PstI digestion of pIG4-5/Phyl and self-ligation to the $X$ XoI site, resulting in removal of $3^{\prime}$ coding sequences. The Sina two-hybrid expression plasmids were derived from pSina/GST by insertion of a fragment containing the entire sina coding sequence into pEG202 and pIG4-5 to make pEG202/Sina and pJG4-5/Sina, respectively. Met-1 of Sina is linked to the twohybrid domains through a nine amino acid linker sequence (FELGTRGSP). All ligation junction sequences and complete sequences of PCR products were confirmed by automated DNA sequencing ( $\mathrm{ABI}$ ).

\section{In vitro binding}

GST fusion proteins were produced in strain HMS174(DE3)pLysS induced with $1 \mathrm{~mm}$ IPTG and grown at $25^{\circ} \mathrm{C}$ for $3 \mathrm{hr}$ in LB medium supplemented with $1 \mathrm{mM} \mathrm{ZnSO}_{4}$. Proteins were purified from whole cell extracts as described (Ausubel et al. 1993) with the addition of a protease inhibitor cocktail $(1 \mu \mathrm{g} / \mathrm{m})$ leupeptin, $1 \mathrm{~mm}$ benzamide, $1 \mu \mathrm{g} / \mathrm{ml}$ pepstatin, $0.2 \mathrm{~mm}$ PMSF $\mid$ to the lysis buffer. Phyl protein was produced by in vitro translation using pET/Phyl with the TNT Coupled Reticulocyte Lysate System (Promega). GST fusion proteins were prebound to glutathione-Sepharose (Pharmacia) such that the concentration of protein on beads was $0.2 \mathrm{mg} / \mathrm{ml}$. The protein-bead complexes were equilibrated in Buffer B [50 mM HEPES (pH 7.4), 120 $\left.\mathrm{mM} \mathrm{NaCl}, 1 \mu \mathrm{M} \mathrm{ZnSO}_{4}, 0.1 \% \mathrm{NP}-40\right]$. For in vitro binding, $12 \mu \mathrm{l}$ of equilibrated protein-bead complex was mixed with $150 \mu \mathrm{l}$ Buffer B, $7.5 \mu \mathrm{l} 10 \mathrm{mg} / \mathrm{ml}$ BSA, and $12 \mu \mathrm{l}$ of reticulocyte translate. The reaction was incubated at $4^{\circ} \mathrm{C}$ for $3 \mathrm{hr}$. Matrices were washed three times with $1 \mathrm{ml}$ of Buffer B prior to electrophoresis and autoradiography.

\section{Yeast two-hybrid association}

Two-hybrid plasmids were transformed into the yeast strain EGY48 and selected as described (Gyuris et al. 1993). For one two-hybrid test, strains were tested for galactose-dependent growth on Leu - plates. For a second test, colonies were grown in liquid glucose/CM Ura $\mathrm{His}^{-} \mathrm{Trp}^{-}$overnight and diluted into galactose $/ \mathrm{CM} \mathrm{Ura}^{-} \mathrm{His}{ }^{-\mathrm{Trp}^{-}}$or glucose/ $\mathrm{CM} \mathrm{Ura}^{-}{ }^{-} \mathrm{His}{ }^{-}$ Trp to achieve log phase growth. $\beta$-Galactosidase activity was quantitatively assayed with ONPG as described (Ausubel et al. 1993).

\section{Acknowledgments}

We are grateful to B. Dickson, M. Fortini, C. Klambt, Z.C. Lai, H. McNeill, M. Mlodzik, G. Rubin, and H. Vaessin for fly stocks; to R. Brent, Z.C. Lai, E. O'Neill, R. Tjian, and H. Vaessin for plasmids. We also thank C. Sullivan for help with binding experiments, T. Harper for assistance with confocal and scanning electron microscopy, and G. Buckles for advice on performing retinal fills. This manuscript was improved by comments from K. Arndt, M. Kuziora, L. Zheng, and A. Slinskey. This work was supported by grants to R.W.C. from the National Institutes of Health (EY10111) and March of Dimes Birth Defects Foundation (Basic Research). R.W.C. is a Pew Scholar in the Biomedical Sciences.
The publication costs of this article were defrayed in part by payment of page charges. This article must therefore be hereby marked "advertisement" in accordance with 18 USC section 1734 solely to indicate this fact.

\section{References}

Allard, J.D., H.C. Chang, R. Herbst, H. McNeill, and M.A. Simon. 1996. The SH2-containing tyrosine phosphatase corkscrew is required during signaling by sevenless, Rasl and Raf. Development 122: 1137-1146.

Ausubel, F.M., R. Brent, R.E. Kingston, D.D. Moore, J.G. Seidman, J.A. Smith, and K. Struhl. 1993. Current Protocols in Molecular Biology. Greene Publishing and John Wiley \& Sons, Inc., New York, NY.

Basler, K., B. Christen, and E. Hafen. 1991. Ligand-independent activation of the sevenless receptor tyrosine kinase changes the fate of cells in the developing Drosophila eye. Cell 64: 1069-1081.

Biggs, W.H., K.H. Zavitz, B. Dickson, A. VanderStraten, D. Brunner, E. Hafen, and S.L. Zipursky. 1994. The Drosophila rolled locus encodes a MAP kinase required in the sevenless signal transduction pathway. EMBO I. 13: 1628-1635.

Brunner, D., K. Ducker, N. Oellers, E. Hafen, H. Scholz, and C. Klambt. 1994a. The ETS domain protein Pointed-P2 is a target of MAP kinase in the Sevenless signal transduction pathway. Nature 370: 386-389.

Brunner, D., N. Oellers, J. Szabad, W.H. Biggs, S.L. Zipursky, and E. Hafen. 1994b. A gain-of-function mutation in Drosophila MAP kinase activates multiple receptor tyrosine kinase signaling pathways. Cell 76: 875-888.

Buckles, G.R., D.J. Smith, and F.N. Katz. 1992. mip causes hyperinnervation of a retinotopic map in Drosophila by excessive recruitment of R7 photoreceptor cells. Neuron 8: 10151029.

Carthew, R.W. and G.M. Rubin. 1990. seven in abstentia, a gene required for specification of $\mathrm{R} 7$ cell fate in the Drosophila eye. Cell 63: 561-577.

Chang, H.C., N.M. Solomon, D.A. Wassarman, F.D. Karim, M. Therrien, G.M. Rubin, and T. Wolff. 1995. phyllopod functions in the fate determination of a subset of photoreceptors in Drosophila. Cell 80: 463-472.

Dickson, B. 1995. Nuclear factors in sevenless signaling. Trends Genet. 11: 106-111.

Dickson, B.J., M. Dominquez, A. van der Straten, and E. Hafen. 1995. Control of Drosophila photoreceptor cell fates by Phyllopod, a novel nuclear protein acting downstream of the Raf kinase. Cell 80: 453-462.

Doc, C.Q., Q. Chu-LaGraff, D.M. Wright, and M.P. Scott. 1991. The prospero gene specifies cell fates in the Drosophila central nervous system. Cell 65: 451-464.

Fortini, M.E., M.A. Simon, and G.M. Rubin. 1992. Signalling by the sevenless protein tyrosine kinase is mimicked by Ras 1 activation. Nature 355: 559-561.

Fortini, M.E., I. Rebay, L.A. Caron, and S. Artavanis-Tsakonis. 1993. An activated Notch receptor blocks cell-fate commitment in the developing Drosophila eye. Nature 365: 555557.

Gyuris, J., E. Golemis, H. Chertkov, and R. Brent. 1993. Cdil, a human G1 and $S$ phase protein phosphatase that associates with Cdk2. Cell 75: 791-803.

Harlow, E. and D. Lane. 1988. Antibodies: A laboratory manual. Cold Spring Harbor Laboratory, Cold Spring Harbor, NY.

Kimmel, B.E., U. Heberlein, and G.M. Rubin. 1990. The home- 
odomain protein rough is expressed in a subset of cells in the developing Drosophila eye where it can specify photoreceptor cell subtype. Genes \& Dev. 4: 712-727.

Lai, Z.C. and G.M. Rubin. 1992. Negative control of photoreceptor development in Drosophila by the product of the yan gene, an ETS domain protein. Cell 70: 609-620.

Lassar, A.B., R.L. Davis, W.E. Wright, T. Kadesch, C. Murre, A. Voronova, D. Baltimore, and H. Weintraub. 1991. Functional activity of myogenic HLH proteins requires hetero-oligomerization with E12/E47-like proteins in vivo. Cell 66: 305315.

Marshall, C.J. 1995. Specificity of receptor tyrosine kinase signaling: transient versus sustained extracellular signal-related kinase activation. Cell 80: 179-185.

Mlodzik, M., Y. Hiromi, U. Weber, C.S. Goodman, and G.M. Rubin. 1990. The Drosophila seven-up gene, a member of the steroid receptor gene superfamily, controls photoreceptor cell fates. Cell 60: 211-224.

Moses, K. and G.M. Rubin. 1991. glass encodes a site-specific DNA-binding protein that is regulated in response to positional signals in the developing Drosophila eye. Genes \& Dev. 5: 583-593.

Mullins, M.C. and G.M. Rubin. 1991. Isolation of temperaturesensitive mutations of the tyrosine kinase receptor sevenless (sev) in Drosophila and their use in determining its time of action. Proc. Natl. Acad. Sci. 88: 9387-9391.

Neufeld, T.P. and G.M. Rubin. 1994. The Drosophila peanut gene is required for cytokinesis and encodes a protein similar to yeast putative bud neck filament proteins. Cell 77:371379.

O'Neill, E.M., I. Rebay, R. Tijan, and G.M. Rubin. 1994. The activities of two Ets-related transcription factors required for Drosophila eye development are modulated by the Ras/ MAPK pathway. Cell 78: 137-147.

Rebay, I. and G.M. Rubin. 1995. Yan functions as a general inhibitor of differentiation and is negatively regulated by activation of the Ras1/MAPK pathway. Cell 81: 857-866.

Rupp, R.A. and H. Weintraub. 1991. Ubiquitous MyoD transcription at the midblastula transition precedes inductiondependent MyoD expression in presumptive mesoderm of $\mathrm{X}$. laevis. Cell 65: 927-937.

Simon, M.A., D.D. Bowtell, G.S. Dodson, T.R. Laverty, and G.M. Rubin. 1991. Ras1 and a putative guanine nucleotide exchange factor perform crucial steps in signaling by the sevenless protein tyrosine kinase. Cell 67: 701-716.

Tomlinson, A. and D.F. Ready. 1987. Cell fate in the Drosophila ommatidium. Dev. Biol. 123: 264-275.

Vaessin, H., E. Grell, E. Wolff, E. Bier, L.Y. Jan, and Y.N. Jan. 1991. prospero is expressed in neuronal precursors and encodes a nuclear protein that is involved in the control of axonal outgrowth in Drosophila. Cell 67: 941-953.

Xu, T. and G.M. Rubin. 1993. Analysis of genetic mosaics in developing and adult Drosophila tissues. Development 117: 1223-1237.

Zheng, L., J. Zhang, and R.W. Carthew. 1995. frizzled regulates mirror-symmetric pattern formation in the Drosophila eye. Development 121: 3045-3055.

Zipursky, S.L. and G.M. Rubin. 1994. Determination of neuronal cell fate: lessons from the R7 neuron of Drosophila. Annu. Rev. Neurosci. 17: 373-397. 


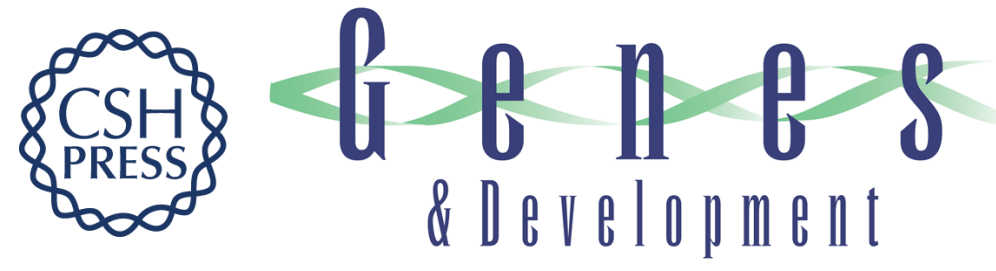

\section{Ras1 signaling and transcriptional competence in the $R 7$ cell of Drosophila.}

R C Kauffmann, S Li, P A Gallagher, et al.

Genes Dev. 1996, 10:

Access the most recent version at doi:10.1101/gad.10.17.2167

References This article cites 31 articles, 6 of which can be accessed free at:

http://genesdev.cshlp.org/content/10/17/2167.full.html\#ref-list-1

License

Email Alerting

Service

Receive free email alerts when new articles cite this article - sign up in the box at the top right corner of the article or click here.

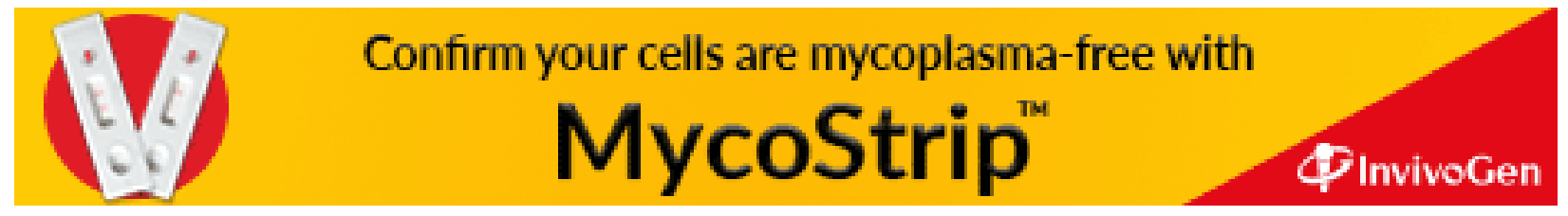

